\title{
Sleep Deprivation Interferes with JAK/ STAT Signaling Pathway and Myogenesis in the Masseter Muscle of Rats
}

\author{
Marina Gomes Galvani Hanna Karen Moreira Antunes Marcos Monico-Neto \\ Veronica Quispe Yujra Carla Maximo Prado Hananiah Tardivo Quintana \\ Flavia de Oliveira Daniel Araki Ribeiro \\ Department of Biosciences, Federal University of São Paulo, UNIFESP, Santos, Brazil
}

\section{Significance of the Study}

- Sleep deprivation activates the Janus kinase/tyrosine kinase-activated transduction factor signaling pathway in the masseter muscle.

- Atrophy was demonstrated in the masseter muscle after sleep deprivation.

- Tissue repair mechanisms are inhibited when stress is not present.

\section{Keywords}

Rat $\cdot$ Masseter $\cdot$ Sleep deprivation - Metyrapone

\begin{abstract}
Objectives: The aim of the study was to study the Janus kinase/tyrosine kinase-activated transduction factor (JAK/ STAT) signaling pathway and myogenesis on the masseter muscle after sleep deprivation and to investigate the role of stress in this scenario. Subjects and Methods: A total of 18 male Wistar rats were divided into the following groups: control $(n=6)$ : animals were not submitted to any procedures, and paradoxical sleep deprivation and vehicle (PSD $+\mathrm{V} ; n=$ 6): animals were subjected to PSD for $96 \mathrm{~h}$ and (PSD + MET; $n=6$ ): animals were subjected to PSD for $96 \mathrm{~h}$ with adminis-
\end{abstract}

tration of metyrapone. Paradoxical sleep deprivation was performed by the modified multiple platforms method. Histopathological analysis, histomorphometry, and immunohistochemistry were performed. Results: The results showed the presence of inflammatory infiltrate in the PSD $+\mathrm{V}$ and PSD + MET groups and atrophy. Histomorphometry showed that the cellular profile area decreased, while cellular density increased in both experimental groups. Expression of $\mathrm{p}$ STAT 3, MyoD, and MyoG increased in the PSD + V group, while the PSD + MET group showed increased expression of IL-6 and p-STAT 3. Conclusion: Our results suggest that sleep deprivation induces an inflammatory response and atrophy in the masseter muscle of rats.

C 2021 The Author(s) Published by S. Karger AG, Basel karger@karger.com www.karger.com/mpp

Karger $\stackrel{\text { ' }}{5}$

GOPEN ACCESS
(C) 2021 The Author(s)

Published by S. Karger AG, Basel

This is an Open Access article licensed under the Creative Commons Attribution-NonCommercial-4.0 International License (CC BY-NC) (http://www.karger.com/Services/OpenAccessLicense), applicable to the online version of the article only. Usage and distribution for commercial purposes requires written permission.
Correspondence to:

Daniel Araki Ribeiro, daribeiro@ unifesp.br 


\section{Introduction}

It has been established that consistent lack of sleep induces cardiovascular disease, dyslipidemia, diabetes, and cognitive impairment $[1,2]$. According to a study by $\mathrm{Me}-$ jri et al. [3], levels of biomarkers for muscle and cardiac injuries increase after sleep deprivation in humans. Monico-Neto et al. [4] reported significant atrophic response in the anterior tibial muscle of rats subjected to the paradoxical sleep deprivation. Similar findings were reported by Dattilo et al. [5]. Sleep deprivation has been shown to cause degradation of muscle protein, which is considered to be an underlying mechanism of atrophy, and also to impair the regeneration of the anterior tibial muscle of rats subjected to cryolysis[6].

Alterations in glucocorticoid secretion have been shown following sleep deprivation or restriction [7]. It would be interesting to know if, and to what extent, sleep loss modulates the levels of some stress hormones as the information will provide insights into the relationship between sleep homeostasis and stress [7]. The model of sleep deprivation in rats by the platform method has been extensively studied in our laboratory as a suitable experimental model of sleep loss $[8,9]$. It uses relatively simple instruments based on muscle atony resulting from entering the rapid eye movement phase to assess the effects of sleep deprivation on health [10].

The Janus kinase/tyrosine kinase-activated transduction factor (JAK/STAT) signaling pathway corresponds to nonreceptor tyrosine kinases found predominantly in the cytoplasm, whose canonical signaling pathway involves the binding of cytokines and hormones to transmembrane receptors [11]. In the skeletal muscle, activation of protein degradation mechanisms via ubiquitin proteasome as well as increased proliferation of satellite cells is triggered by the binding of IL- 6 or TGF- $\beta$ to their specific receptors [12]. Following the canonical pathway, p-STAT 3 dimers translocate to the nucleus and trigger the above effects. IL- 6 is a cytokine involved in immune responses, inflammatory processes, and hematopoiesis [13]. Physical exercise stimulates the release of IL-6 in skeletal muscles favoring hypertrophy [14]. Pelosi et al. [15] found that pharmacological inhibition of IL-6 improved the dystrophic phenotype of Duchenne muscular dystrophy by reducing the damage caused by the catabolic environment present in disease.

With regard to muscle regeneration, some stimuli, such as physical trauma or growth factors, are able to disrupt the quiescence state and thus restart the cell cycle, expressing some myogenic factors like MyoD and myogenin [16]. Recently, it was also found that muscle regeneration and autophagy were triggered during sleep deprivation in the rat masseter muscle by the expression of MyoD/myogenin and LC3B, respectively [8]. However, further studies are needed to clarify the impact of stress in this context. The aim of this study was to investigate the JAK/STAT signaling pathway and myogenesis after paradoxical sleep deprivation as well as the role of stress triggered by activation of the HPA axis in masseter muscle of Wistar rats.

\section{Material and Methods}

Animals and Ethical Procedures

Male Wistar EPM-1 rats, 12 weeks old and approximately 300 $350 \mathrm{~g}$ of body mass, were used from the Federal University of Sao Paulo, UNIFESP. Throughout the experimental period, the rats remained under controlled temperature $\left(22 \pm 2^{\circ} \mathrm{C}\right)$, relative humidity ( $55 \pm 5 \%)$, active exhaustion, 12 -h light/dark cycle $(7 / 19 \mathrm{~h})$, receiving filtered water, and Nuvital ${ }^{\circledR}$ feed without restriction. The study was approved by the Animal Ethics Committee (No. 4569140818).

\section{Paradoxical Sleep Deprivation and Metyrapone}

Administration

The animals from experimental groups were submitted to paradoxical sleep deprivation (PSD) for $96 \mathrm{~h}$. Selective deprivation of paradoxical sleep was performed by using the modified multiple platform method. This protocol promotes approximately $90 \%$ paradoxical sleep loss [10]. The animals from the control (CTRL) group were kept in housing cages in the same room, during the same period where the PSD protocols were performed in the experimental groups.

A total of 18 animals were randomly distributed into 3 groups: CTRL ( $n=6$; animals were not submitted to any procedures); PSD and vehicle deprivation (PSD $+\mathrm{V} ; n=6$; animals were subjected to PSD for $96 \mathrm{~h}$ with administration of saline in propylene glycol), and PSD associated with metyrapone administration (PSD + MET; $n=6 ; 100 \mathrm{mg} / \mathrm{kg}$ ). Metyrapone was administered at a dose of 100 $\mathrm{mg} / \mathrm{kg}$ in a volume of $1 \mathrm{~mL} / \mathrm{kg}$ 2-methyl-1,2-di-3-pyridyl-1-propanone (metyrapone, Sigma Aldrich, St. Louis, MO, USA) intraperitoneally. Both vehicle solution and metyrapone solution were administered from 12 to $12 \mathrm{~h}$, in order to respect its metabolization time.

\section{Histopathological Analysis}

After euthanasia, dissection of the masseter muscles was immediately performed, subsequently fixed in $10 \%$ buffered formalin for $24 \mathrm{~h}$ for histological processing. The histological procedure was performed in $4-\mu \mathrm{m}$-thick sections, stained in HE. The following parameters were evaluated: quantity and shape of myocytes, evaluation of cell nuclei, presence of inflammatory infiltrate, and atypical cells. 


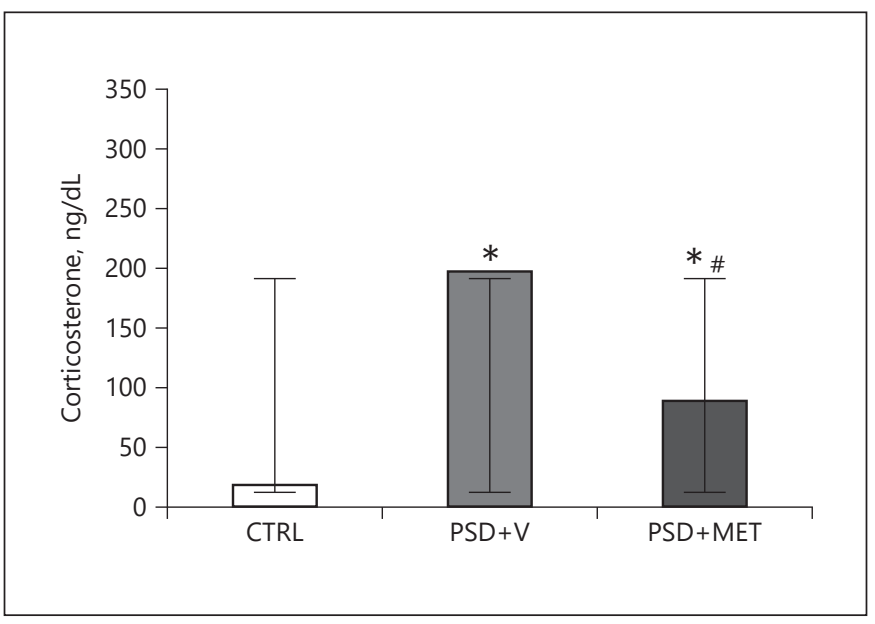

Fig. 1. Plasma corticosterone concentrations. ${ }^{*} p<0.05$ compared to CTRL and ${ }^{\#} p<0.05$ compared to PSD + V. CTRL, control; PSD $+\mathrm{V}$, paradoxical sleep deprivation and vehicle; PSD + MET, paradoxical sleep deprivation and metyrapone.

\section{Histomorphometric Analysis: Cell Profile Area and Density}

From the HE-stained slides, a cut of each animal was chosen and 3 fields were photographed with a $\times 40$ objective. A test system (frame) of a known area of $40,000 \mu \mathrm{m}^{2}(200 \times 200$ $\mu \mathrm{m})$ was selected. These values were converted to $0.04 \mathrm{~mm}^{2}$ $(0.2 \times 0.2 \mathrm{~mm})$. The sides of the frame had 2 continuous lines (forbidden lines) and 2 dotted lines (allowed lines). For cell density (number of cells $/ \mathrm{mm}^{2}$ ), the fibers within the frame were counted so that the fibers tangent to the forbidden line were not counted and those tangent to the dotted line entered the count. The cell profile area $\left(\mu \mathrm{m}^{2}\right)$ and cell density were accounted for using 3 cuts per animal and 3 fields. For these analyses, the inverted Axio Observer.D1 Zeiss ${ }^{\circledR}$ microscope coupled to Axio Vision 4.8 software was used.

\section{Immunohistochemistry}

Cytoplasmic Expression

For the evaluation of expression of IL-6, JAK 2, STAT 3 , p-STAT 3, and SOCS 3 proteins, $3-\mu \mathrm{m}$ serial sections were pretreated with citric acid buffer (10 nM, pH 6.1, $0.1 \mathrm{M}$ of citric acid, Synth ${ }^{\circledR}$, São Paulo, Brazil) in microwaves for 3 cycles of $5 \mathrm{~min}$ each for antigen retrieval. The sections were then incubated with $0.3 \%$ hydrogen peroxide for inactivation of endogenous peroxidase and for the specific protein blocking step, the Starr Trek Universal HRP Detection Kit (Biocare Medical ${ }^{\circledR}$, Pacheco, CA, USA) was used for $30 \mathrm{~min}$, followed by 2 washes with PBS.

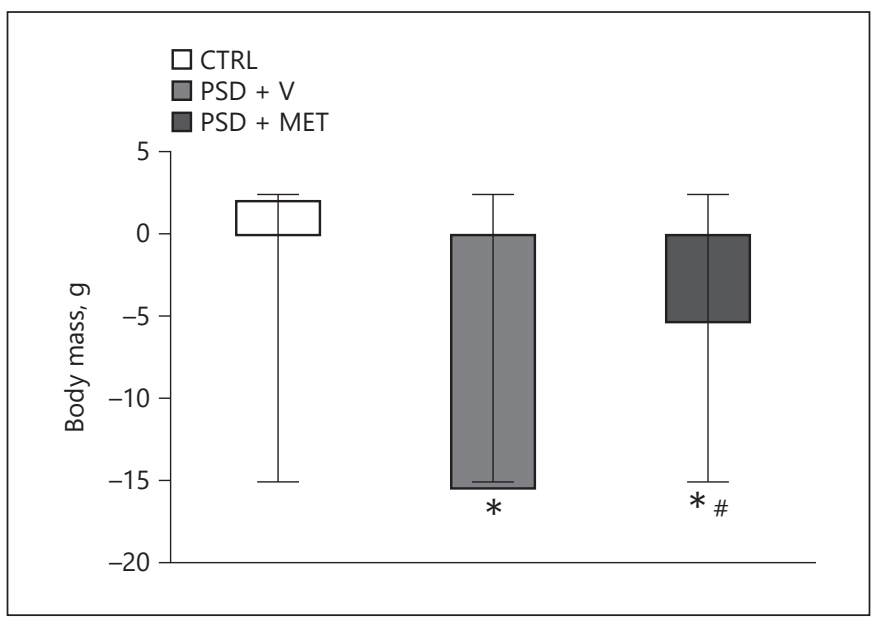

Fig. 2. Body mass from rats submitted to sleep deprivation and treatment with metyrapone. ${ }^{*} p<0.05$ compared to CTRL and ${ }^{\#} p<0.05$ compared to PSD + V. CTRL, control; PSD + V, paradoxical sleep deprivation and vehicle; PSD + MET, paradoxical sleep deprivation and metyrapone.

Primary antibodies, all obtained from Santa Cruz Biotechnology Inc ${ }^{\circledR}$ (Santa Cruz, CA, USA), were used as follows: IL-6 at 1:150 dilution, JAK-2 at a ratio of 1:200, STAT 3 diluted 1:100, p-STAT 3 at 1:200, and SOCS 3 at 1:200 deposited on slides and incubated overnight at $4^{\circ} \mathrm{C}$. The specimens were then subjected to 2 washes with PBS and incubated with biotinylated secondary antibody for 30 min (Starr Trek Universal HRP Detection Kit, Biocare Medical $^{\circledR}$, Pacheco, CA USA), washed with PBS, incubated with streptavidin conjugated with hydrogen peroxide also for $30 \mathrm{~min}$, and then stained with DAB (3,3-diaminobenzidine, $0.05 \%$ - DAKO North America Inc. ${ }^{\circledR}$, Carpinteria, CA, USA). Counterstaining was performed using Harris hematoxylin (Sigma ${ }^{\circledR}$, St. Louis, MO, USA). Once the marking was performed, 1,000 muscle cells were evaluated in $\times 400$ magnification, by the systematic randomization system.

\section{Nuclear Expression}

For immunohistochemical evaluation of MyoD and MyoG, the same protocol for cytoplasmic expression antibodies described earlier was used, with a dilution of 1:100 for both immunomarkers. The density of immunoreactive nuclei was determined using three $0.04-\mathrm{mm}^{2}$ area fields per animal, photographed from the Axio Observer inverted microscope Zeiss ${ }^{\circledR}$ D1 (Darmstadt, Germany) coupled with Axio Vision 4.8 software, in $\times 1,000$ magnification and nuclei positively marked by the immunohistochemistry reaction. 


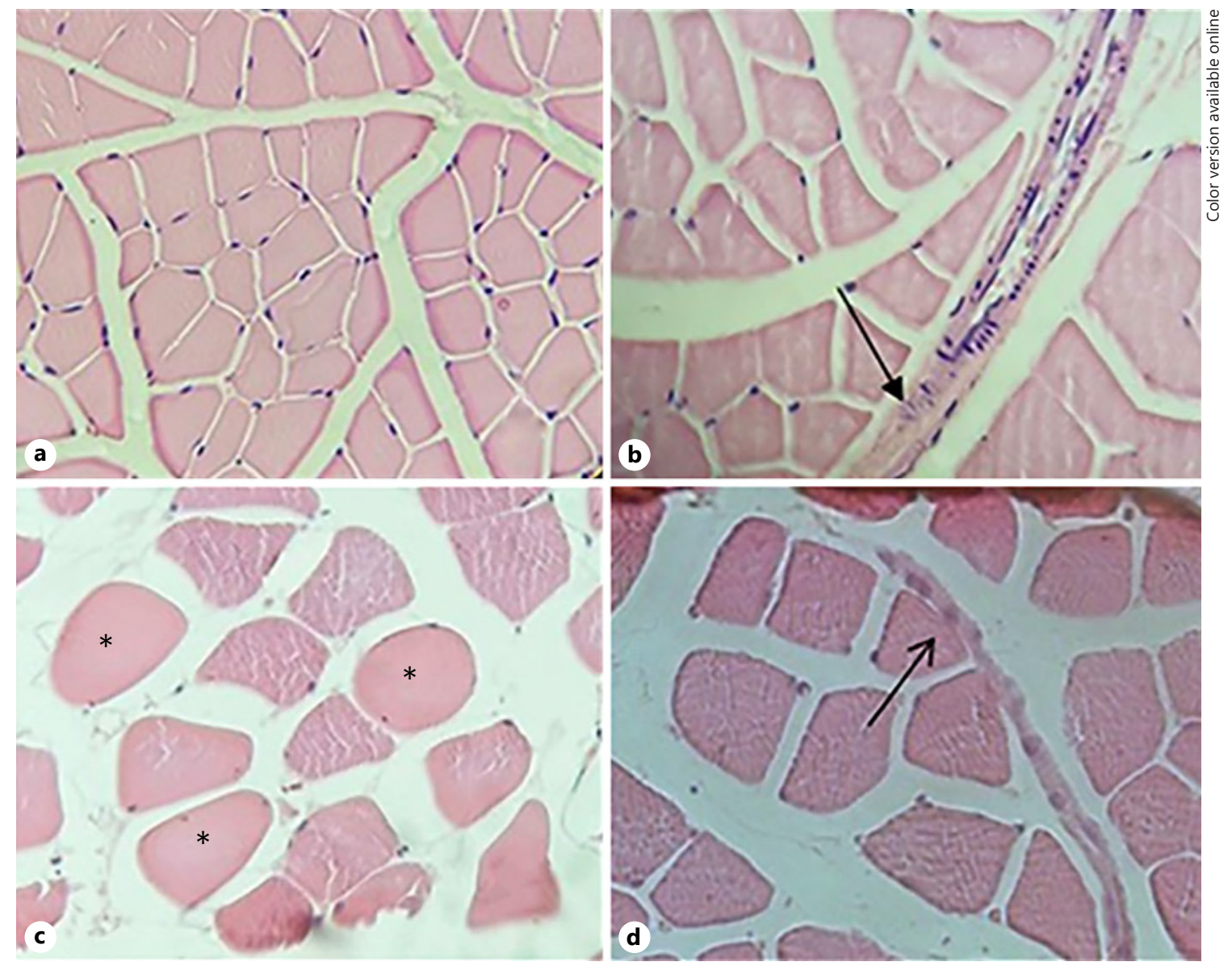

Fig. 3. Photomicrographs from masseter muscle of rats submitted to sleep deprivation and treated with metyrapone. a The CTRL group masseter muscle tissue with preserved architecture is observed. $\mathbf{b}$ PSD + V group with inflammatory infiltrate pointed by black arrow. c PSD + MET group. The black asterisks show overlapping cells. PSD + MET group. $\mathbf{d}$ Inflammatory infiltrate pointed by black arrow. HE stain, $\times 40$ magnification. PSD + MET, paradoxical sleep deprivation and metyrapone.

\section{Statistical Analysis}

Results were expressed as mean \pm standard deviation. The Kruskal-Wallis test was used, followed by Dunn's multiple comparisons test. Statistical calculations were performed using GraphPad Prism version 5.0 software, and a significance level of $95 \%(p<0.05)$ was adopted.

\section{Results}

Plasma Corticosterone Concentration and Body Mass Plasma corticosterone concentration increased in the $\mathrm{PSD}+\mathrm{V}(p<0.05)$ and PSD $+\mathrm{MET}(p<0.05)$ groups compared to the CTRL group. However, the PSD + MET group showed a statistically significant reduction $(p<$ $0.05)$ compared to PSD $+V$. Figure 1 shows the numerical results.

Body mass also showed a significant reduction in both experimental groups when compared to CTRL $(p<0.05)$. The PSD + MET group showed lower mass reduction than PSD + V ( $p<0.05)$ (Fig. 2).

\section{Histopathological Analysis}

The masseter muscle from the CTRL group was identified as multinucleated polygonal myocytes without inflammatory infiltrate with peripheral nuclei (Fig. 3a). On the other hand, the PSD + V group showed evident pathological alterations, such as the presence of mild inflammatory infiltrate in the perimysium, as 


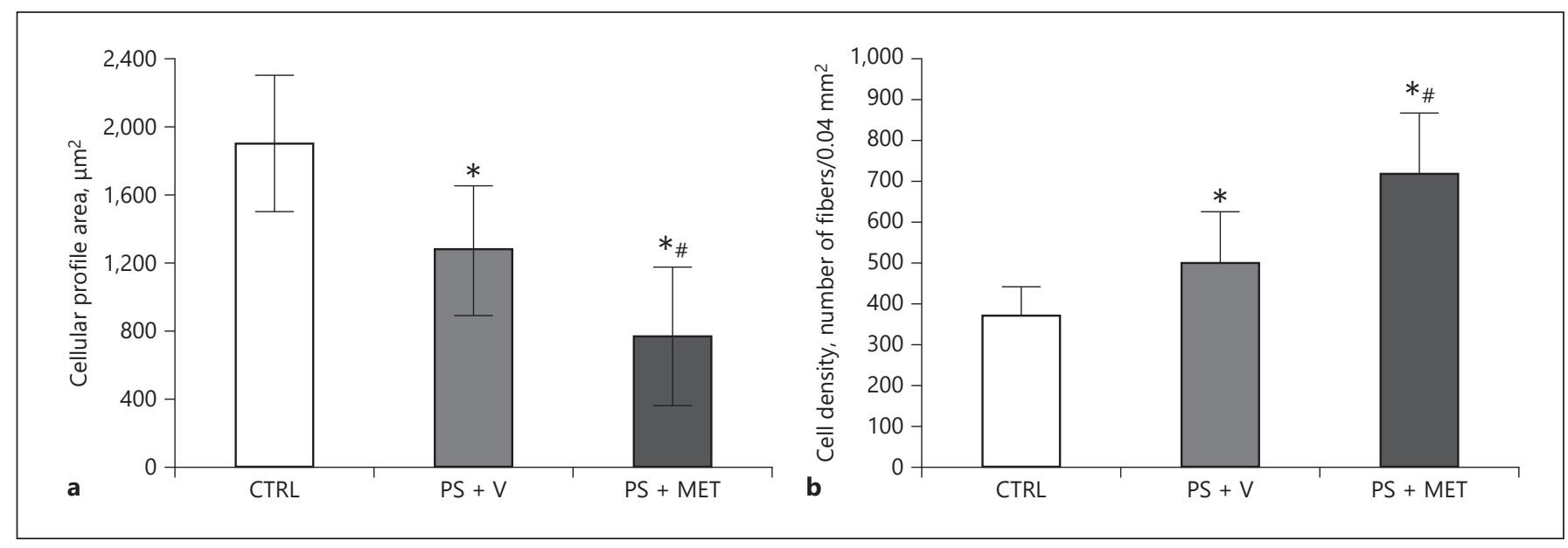

Fig. 4. Cellular profile area (a) and cell density (b) from masseter muscle of rats submitted to sleep deprivation and treated with metyrapone. Results presented as mean and SD. ${ }^{*} p<0.05$ when compared to CTRL; ${ }^{\#} p<0.05$ when compared to the PSD + V group. CTRL, control group; PSD + V, paradoxical sleep deprivation and vehicle; PSD + MET, paradoxical sleep deprivation and metyrapone.

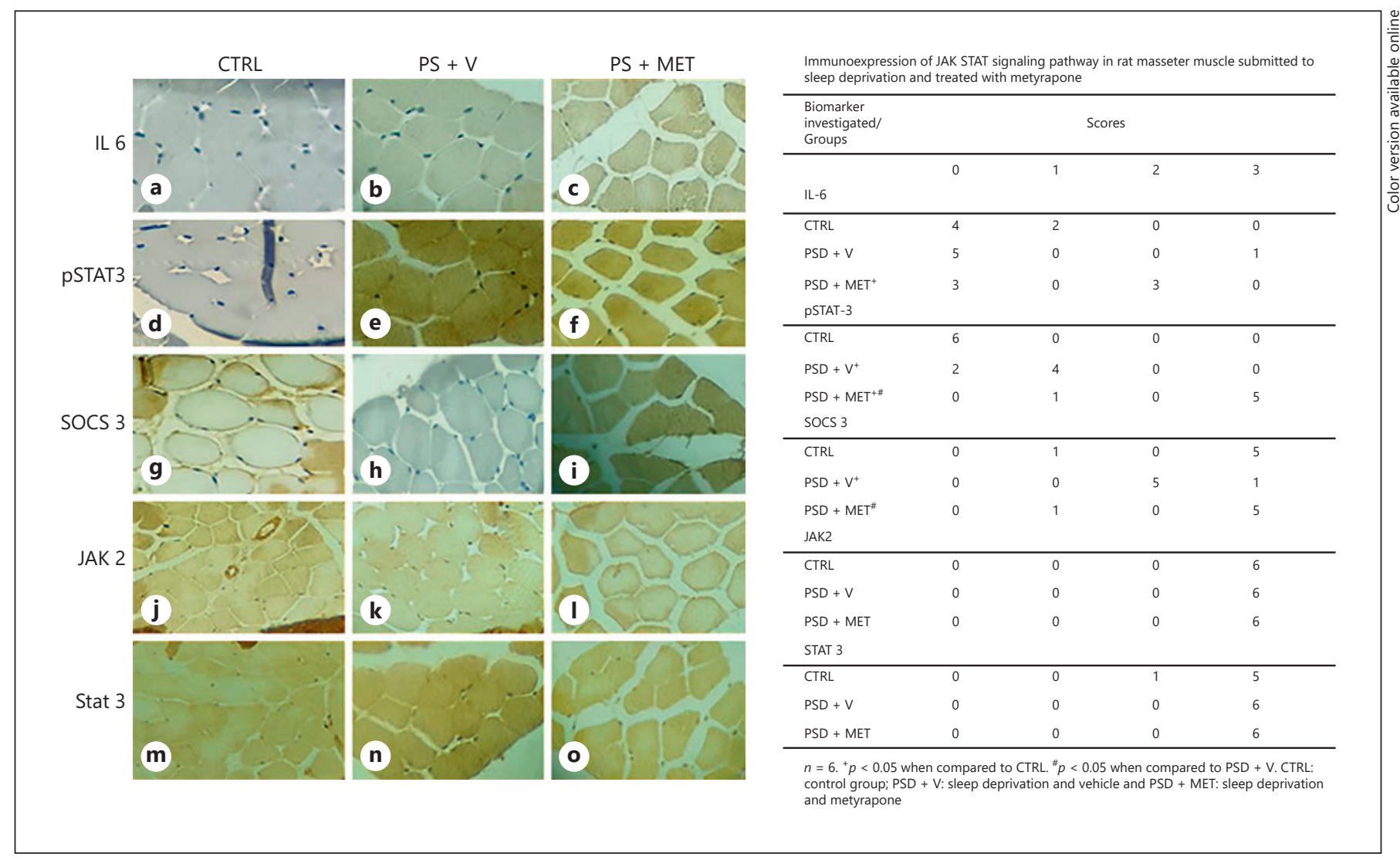

Fig. 5. Immunohistochemistry for JAK/STAT signaling pathway in rat masseter muscle of rats submitted to sleep deprivation and treated with metyrapone. Immunohistochemistry stain, $\times 40$ magnification. a-c Immunohistochemistry for IL-6. d-f Immunohistochemistry for pSTAT3. g-i Immunohistochemistry for SOCS3. j-I Immunohistochemistry for JAK2. m-o Immunohistochemsitry for STAT3. JAK, Janus kinase; STAT, tyrosine kinaseactivated transduction factor. 


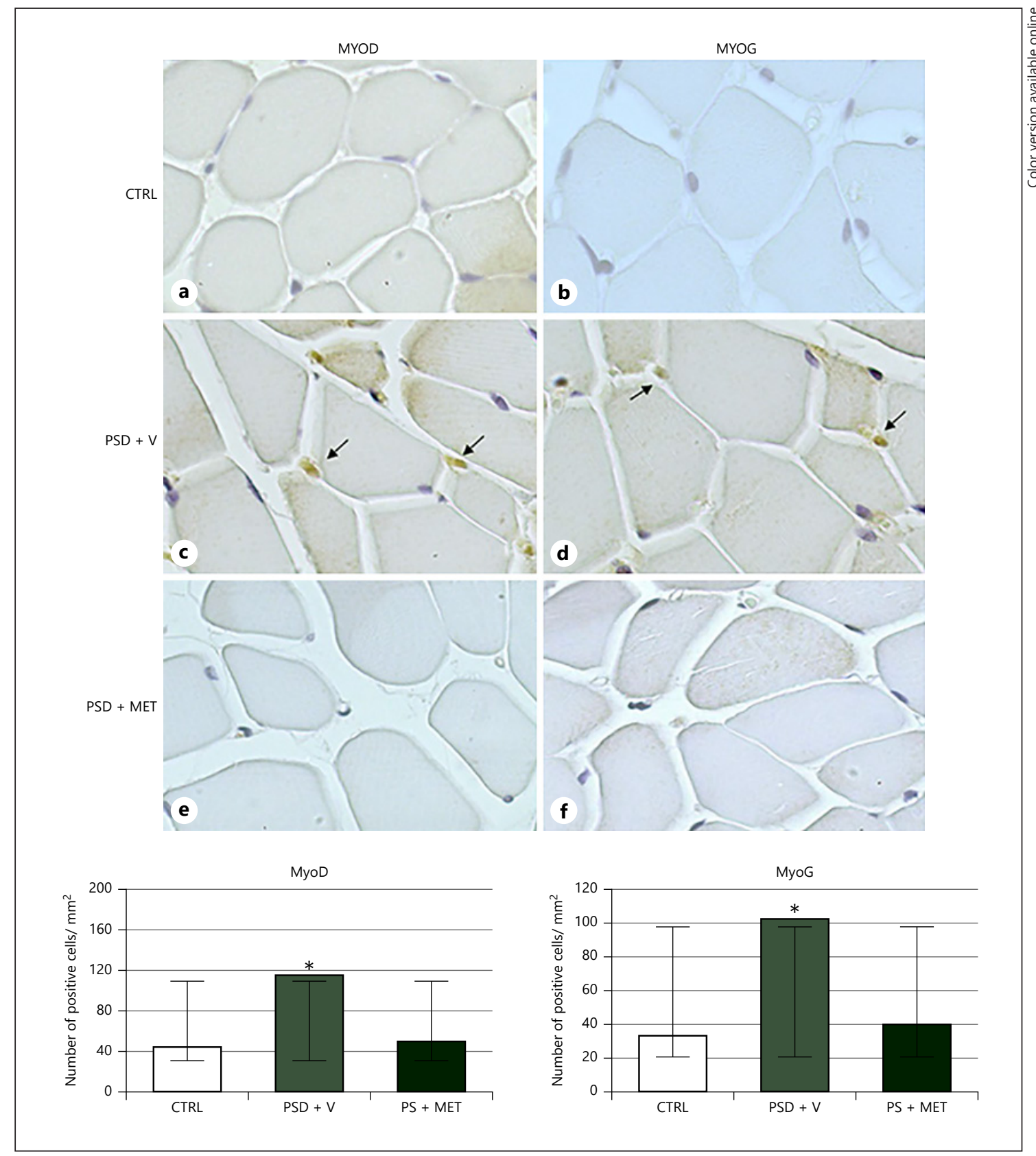

Fig. 6. Immunohistochemistry for MyoD and myogenin in rat masseter muscle of rats submitted to sleep deprivation and treated with metyrapone. Note the nuclear staining (arrows). Immunohistochemistry stain, $\times 40$ magnification. a, b CTRL group. c, d PSD + V group. e, f PSD + MET. Cellular profile area and cell density from masseter muscle of rats submitted to sleep deprivation and treated with metyrapone. Results presented as mean and SD. ${ }^{*} p<0.05$ when compared to CTRL. CTRL, control group; PSD + V, paradoxical sleep deprivation and vehicle; PSD + MET, paradoxical sleep deprivation and metyrapone.

depicted by the presence of neutrophils cells, besides high cellularity (Fig. 3b). In the PSD + MET group, the presence of inflammatory infiltrate was also observed in the perimysium, as well as the presence of polymor- phonuclear inflammatory cells (Fig. 3d). Hypercellular cells were also found by the intense hyaline aspect (Fig. 3c). 


\section{Morphometric Analysis}

The analysis related to the cell profile area showed significant reduction in both the PSD $+\mathrm{V}(p<0.05)$ and the PSD + MET groups $(p<0.05)$ when compared to the CTRL group. In addition, the results revealed that the PSD + MET group was significantly smaller $(p<0.05)$ than the PSD $+V$ group. Cell density showed a significant increase in the PSD $+\mathrm{V}$ group $(p<0.05)$ when compared to the CTRL group. Similarly, the PSD + MET group increased significantly $(p<0.05)$ when compared with the PSD $+V$ group. The results are shown in Figure 4.

\section{Cytoplasmic Expression}

Investigation of the JAK 2/STAT 3 pathway by immunohistochemistry revealed that expression of IL- 6 was significantly higher in the PSD + MET group $(p<0.05)$ than the CTRL group. Also, the PSD + MET group had significantly higher p-STAT 3 expression $(p<0.05)$ than both the CTRL and the PSD + V groups. There was no significant difference $(p<0.05)$ in the expression of SOCS 3 in the PSD + MET group when compared to the CTRL group, but there was a significant increase $(p<0.05)$ compared to the PSD $+V$ group. However, the PSD $+V$ group showed a statistically significant difference $(p<0.05)$ in relation to the CTRL group. Finally, the expression of both JAK 2 and p-STAT 3 in the experimental groups was not significantly different $(p<0.05)$ for both CTRL and experimental groups. Such findings are demonstrated in Figure 5.

\section{Nuclear Expression}

Regarding the results of nuclear expression of MyoD and MyoG, the PSD + V group showed a significant increase $(p<0.05)$ in relation to both markers. However, the PSD + MET group did not show significant statistical differences $(p>0.05)$ related to these markers when compared to the CTRL and sleep-deprived groups. The results are demonstrated in Figure 6.

\section{Discussion}

This study evaluated the effects of sleep deprivation in the presence of stress on the masseter muscle of Wistar rats. For this purpose, one of the experimental groups received the drug metyrapone, which is known to suppress the HPA axis, a pathway activated in response to stressful events from several sources in the environment [17].

The significant increase in plasma corticosterone in the PSD + MET group allows us to affirm that it did not completely inhibit the synthesis of this hormone. However, when there is a comparison between the experimental groups, levels of plasma corticosterone in the group receiving metyrapone decreased significantly, confirming the action of the drug and validating its use in this study in terms of the isolation of stress.

In this study, the presence of polymorphonucleated cell infiltrates in both the PSD $+V$ and PSD + MET groups indicates the presence of acute inflammatory response. Also, the presence of IL- 6 corroborates the study that sleep deprivation is closely associated with the release of this mediator [18], mainly due to its significant detection in the PSD + MET group. Irwin et al. [19] assessed the impact of this condition on gene expression, and the results exhibited elevated expression of TNF- $\alpha$ in individuals suffering partial sleep deprivation; interestingly, the significant cytoplasmic increase in this cytokine (TNF- $\alpha$ ) in the PSD + MET group demonstrates the pathological effect of sleep restriction without stress interference. IL-6 is stimulated by physical exercise, favoring muscle hypertrophy [20]. However, high plasma levels of this cytokine are capable of inducing muscle damage [21]. Previous studies conducted by our group have confirmed these findings in masseter of rats submitted to sleep deprivation $[8,9]$.

The JAK/STAT 3 pathway is classically activated by binding IL-6 to its Gp 130 or interleukin-like receptors being able to contribute to promoting protein degradation by signaling myostatin [22]. Bonetto et al. [23] have postulated that suppression of this pathway inhibits muscle degradation in cancer-related cachexia. In addition, some authors have suggested that activation of the JAK/ STAT signaling pathway is correlated with myogenic differentiation [24]. Based on these findings, the nuclear translocation of STAT 3 appears to be able to increase myogenin expression [25]. Thus, the detection of p-STAT 3 in the PSD + V group, together with the significant decrease in SOCS 3 , indicates the activation of the pathway in this group, which can also be related to the biological response to muscular atrophy, as depicted by histomorphometric analysis. Such findings were also confirmed by high nuclear expression of both MyoD and MyoG in the PSD $+\mathrm{V}$ group, indicating the onset of cell regeneration processes followed by sleep-induced muscle damage. Finally, the absence of significant differences in expression of JAK 2 and STAT 3 is related to the physiological presence of these participants from several signaling pathways involving JAKs and STATs [26].

Despite the deleterious effects of cortisol found in skeletal striated muscle, the stress response plays important 
role in adapting to harmful conditions [27]. Thus, its absence, caused by the administration of metyrapone, associated with the experimental sleep deprivation, could indicate that the organism as a whole was not able to trigger muscle repair induced by sleep deprivation. It was also confirmed by the absence of significant expression of MyoD and MyoG in the sleep-deprived group associated with metyrapone. Additionally, this scenario resembles the stress "depletion phase" as the defenses fail to re-establish homeostasis, leading to the first signs and symptoms of illness.

\section{Conclusion}

Our results are consistent with notion that sleep deprivation induced inflammatory response and atrophy in the masseter muscle of rats. In this regard, the mechanisms related to the effects of sleep deprivation must be continually studied in order to develop methods and therapies to minimize or even suppress them, since it is a condition widely observed in the current society.

\section{Statement of Ethics}

This study was approved by the Animal Ethics Committee of the Federal University of São Paulo, UNIFESP (No. 4569140818).

\section{Conflict of Interest Statement}

All authors declare that there is no conflict of interest.

\section{Funding Sources}

M.G.G. was a recipient from CAPES (Coordenação de Apoio de Pessoal de Nivel Superior, master level, Grant number \#001). V.Q.Y., M.M.N., H.K.M.A., C.M.P., and D.A.R. are recipients of a grant from $\mathrm{CNPq}$ (Conselho Nacional de Desenvolvimento Cientifico e Tecnologico, Grant Number \#001).

\section{Author Contributions}

Conception of the project: M.G.G., V.Q.Y., H.K.M.A., and D.A.R. Experimental design: M.G.G., H.K.M.A., M.M.N., and C.M.P. Data interpretation: all authors. Writing the manuscript: all authors.

\section{References}

1 Liu H, Chen A. Roles of sleep deprivation in cardiovascular dysfunctions. Life Sci. 2019; 219:231-7.

2 Lyall LM, Wyse CA, Graham N, Ferguson A, Lyall DM, Cullen B, et al. Association of disrupted circadian rhythmicity with mood disorders, subjective wellbeing, and cognitive function: a cross-sectional study of 91105 participants from the UK Biobank. Lancet Psychiatry. 2018;5:507-14.

3 Mejri MA, Yousfi N, Hammouda O, Tayech A, Ben Rayana MC, Driss T, et al. One night of partial sleep deprivation increased biomarkers of muscle and cardiac injuries during acute intermittent exercise. J Sports Med Phys Fitness. 2017;57(5):643-51.

4 Monico-Neto M, Giampá SQ, Lee KS, de Melo CM, Souza Hde S, Dáttilo M, et al. Negative energy balance induced by paradoxical sleep deprivation causes multicompartmental changes in adipose tissue and skeletal muscle. Int J Endocrinol. 2015:908159.

5 Dattilo M, Antunes HK, Medeiros A, Mônico-Neto M, Souza HS, Lee KS, et al. Paradoxical sleep deprivation induces muscle atrophy. Muscle Nerve. 2012;45(3):431-3.

6 Monico-Neto M, Dáttilo M, Ribeiro DA, Lee KS, de Mello MT, Tufik S, et al. REM sleep deprivation impairs muscle regeneration in rats. Growth Factors. 2017;35:12-8.
7 Machado RB, Hipólide DC, Benedito-Silva AA, Tufik S. Sleep deprivation induced by the modified multiple platform technique: quantification of sleep loss and recovery. Brain Res. 2004;1004(1-2):45-51.

8 Yujra VQ, Moreira Antunes HK, Mônico区 Neto M, Quintana HT, Oliveira F, Galvani MG, et al. Paradoxical sleep deprivation induces differential biological response in rat masticatory muscles: inflammation, autophagy and myogenesis. J Oral Rehabil. 2020; 47(3):289-300.

9 Yujra VQ, Antunes HKM, Mônico-Neto M, Pisani LP, Santamarina AB, Quintana HT, et al. Sleep deprivation induces pathological changes in rat masticatory muscles: role of toll like signaling pathway and atrophy. J Cell Biochem. 2018;119(2):2269-77.

10 Arrigoni E, Chen MC, Fuller PM. The anatomical, cellular and synaptic basis of motor atonia during rapid eye movement sleep. J Physiol. 2016;594(19):5391-414.

11 Qian CJ, Yao J, Si JM. Nuclear JAK2: form and function in cancer. Anat Rec. 2011;294(9): 1446-59.

12 Guadagnin E, Mázala D, Chen YW. STAT3 in skeletal muscle function and disorders. Int J Mol Sci. 2018;19(8):2265.

13 Hirano T. Interleukin 6 and its receptor: ten years later. Int Rev Immunol. 1998;16(3-4): 249-84.
14 Peake JM, Della Gatta P, Suzuki K, Nieman DC. Cytokine expression and secretion by skeletal muscle cells: regulatory mechanisms and exercise effects. Exerc Immunol Rev. 2015;21:8-25.

15 Pelosi L, Berardinelli MG, de Pasquale L, Nicoletti C, D'Amico A, Carvello F, et al. Functional and morphological improvement of dystrophic muscle by interleukin 6 receptor blockade. EBioMedicine. 2015;2(4):285-93.

16 Dumont NA, Bentzinger CF, Sincennes MC, Rudnicki MA. Satellite cells and skeletal muscle regeneration. Compr Physiol. 2015;5(3): 1027-59.

17 Herman JP, McKlveen JM, Ghosal S, Kopp B, Wulsin A, Makinson R, et al. Regulation of the hypothalamic-pituitary-adrenocortical stress response. Compr Physiol. 2016;6(2):603-21.

18 Yehuda S, Sredni B, Carasso RL, KenigsbuchSredni D. REM sleep deprivation in rats results in inflammation and interleukin-17 elevation. J Interferon Cytokine Res. 2009;29(7): 393-8.

19 Irwin MR, Wang M, Campomayor CO, Collado-Hidalgo A, Cole S. Sleep deprivation and activation of morning levels of cellular and genomic markers of inflammation. Arch Intern Med. 2006;166(16):1756-62. 
20 Lightfoot AP, Cooper RG. The role of myokines in muscle health and disease. Curr Opin Rheumatol. 2016;28(6):661-6.

21 Zhou J, Liu B, Liang C, Li Y, Song YH. Cytokine signaling in skeletal muscle wasting. Trends Endocrinol Metab. 2016;27(5):335-47.

22 Lei CT, Su H, Ye C, Tang H, Gao P, Wan C, et al. The classic signalling and trans-signalling of interleukin-6 are both injurious in podocyte under high glucose exposure. J Cell Mol Med. 2018;22(1):251-60.
23 Bonetto A, Aydogdu T, Jin X, Zhang Z, Zhan $\mathrm{R}$, Puzis L, et al. Jak/STAT3 pathway inhibition blocks skeletal muscle wasting downstream of IL- 6 and in experimental cancer cachexia. Am J Physiol Endocrinol Metab. 2012; 303(3):E410-21.

$24 \mathrm{Wu} \mathrm{Z}$, Woodring PJ, Bhakta KS, Tamura K, Wen F, Feramisco JR, et al. p38 and extracellular signal-regulated kinases regulate the myogenic program at multiple steps. Mol Cell Biol. 2000;20(11):3951-64.
25 Guillet-Deniau I, Burnol AF, Girard J. Identification and localization of a skeletal muscle secrotonin 5-HT2A receptor coupled to the Jak/STAT pathway. J Biol Chem. 1997; 272(23):14825-9.

26 Darnell JE Jr. STATs and gene regulation. Science. 1997;277(5332):1630-5.

27 Selye H. A syndrome produced by diverse nocuous agents. Nature. 1936;138(3479):32. 